\title{
Evidence for non-isotropic emitter
}

orientation in a red phosphorescent organic light-emitting diode and its implications for determining the emitter's radiative quantum efficiency

Cite as: Appl. Phys. Lett. 99, 163302 (2011); https://doi.org/10.1063/1.3653475

Submitted: 07 September 2011 . Accepted: 29 September 2011 . Published Online: 18 October 2011

Tobias D. Schmidt, Daniel S. Setz, Michael Flämmich, Jörg Frischeisen, Dirk Michaelis, Benjamin C. Krummacher, Norbert Danz, and Wolfgang Brütting

\section{ARTICLES YOU MAY BE INTERESTED IN}

Determination of molecular dipole orientation in doped fluorescent organic thin films by photoluminescence measurements

Applied Physics Letters 96, 073302 (2010); https://doi.org/10.1063/1.3309705

Light extraction and optical loss mechanisms in organic light-emitting diodes: Influence of the emitter quantum efficiency

Journal of Applied Physics 104, 123109 (2008); https://doi.org/10.1063/1.3043800

Comparing the emissive dipole orientation of two similar phosphorescent green emitter molecules in highly efficient organic light-emitting diodes

Applied Physics Letters 101, 253304 (2012); https://doi.org/10.1063/1.4773188

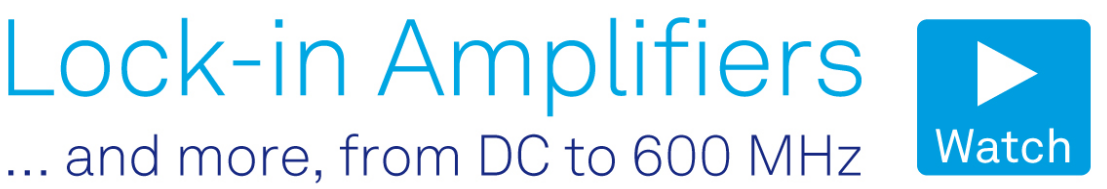




\title{
Evidence for non-isotropic emitter orientation in a red phosphorescent organic light-emitting diode and its implications for determining the emitter's radiative quantum efficiency
}

\author{
Tobias D. Schmidt, ${ }^{1, a)}$ Daniel S. Setz, ${ }^{2}$ Michael Flämmich, ${ }^{3}$ Jörg Frischeisen, ${ }^{1}$ \\ Dirk Michaelis, ${ }^{3}$ Benjamin C. Krummacher, ${ }^{2}$ Norbert Danz,, ${ }^{3}$ and Wolfgang Brütting ${ }^{1, b)}$ \\ ${ }_{1}^{1}$ Institute of Physics, University of Augsburg, 86135 Augsburg, Germany \\ ${ }^{2}$ OSRAM Opto Semiconductors, OLED-Lighting, 93055 Regensburg, Germany \\ ${ }^{3}$ Fraunhofer Institute for Applied Optics and Precision Engineering, 07745 Jena, Germany
}

(Received 7 September 2011; accepted 29 September 2011; published online 18 October 2011)

\begin{abstract}
The efficiency of organic light-emitting diodes is limited as only a fraction of the consumed electrical power is converted into light that is finally extracted to air. Especially, the radiative quantum efficiency of the guest-host system is of interest and should be close to unity to achieve highly efficient devices. We show that the red phosphorescent emitter $\operatorname{Ir}(\mathrm{MDQ})_{2}$ (acac) doped in an $\alpha$-NPD matrix exhibits a profound non-isotropic dipole orientation. Ignoring this feature leads to a significant overestimation of the emitter efficiency. Furthermore, we demonstrate the huge potential for efficiency enhancement of mainly parallel emitter orientation in phosphorescent organic light-emitting diodes. (C) 2011 American Institute of Physics. [doi:10.1063/1.3653475]
\end{abstract}

In recent years, the efficiency of organic light-emitting diodes (OLEDs) has been increasing continuously, and first commercial products with acceptable luminous efficacy in general lighting have been presented. In terms of device physics, not the luminous efficacy but the external quantum efficiency (EQE) is of primary interest. The EQE of an OLED is given by ${ }^{1}$

$$
\mathrm{EQE}=\gamma \cdot \eta_{\mathrm{s} / \mathrm{t}} \cdot q_{\mathrm{eff}}(q) \cdot \eta_{\mathrm{out}},
$$

where $\gamma$ is the charge carrier balance and $\eta_{\mathrm{s} / \mathrm{t}}$ represents the fraction of excitons that is allowed to decay radiatively by spin statistics. $q_{\text {eff }}$ is the effective radiative quantum efficiency of the emitter material, with $q$ being its intrinsic value in the absence of an OLED cavity. ${ }^{2}$ Finally, $\eta_{\text {out }}$ is the outcoupling factor that is mainly determined by the optical environment, i.e., the refractive indices and thicknesses of the used layers in the device, and the orientation of the lightemitting molecules. It is important to note that both $q_{\mathrm{eff}}$ and $\eta_{\text {out }}$ are influenced by the OLED cavity; the former via the radiative lifetime of the emitter and the latter through redistribution of energy between different modes of the cavity. Furthermore, the coupling of radiating dipoles to stratified media is known to depend on the orientation of their transition dipole moment with respect to the layer stack, with vertically oriented dipoles being particularly non-favorable for device efficiency, as they dissipate their energy almost completely to surface plasmons at metallic electrodes. ${ }^{3}$

It was shown recently that non-isotropic emitter orientation, a well known feature of polymeric OLEDs, ${ }^{4,5}$ may also play a significant role in devices based on small molecules and particularly in dye-doped guest-host systems. After the initial demonstration of this effect for fluorescent emitters, ${ }^{6,7}$ it has also been shown that even phosphorescent emitter molecules doped in isotropic matrices can exhibit a predomi-

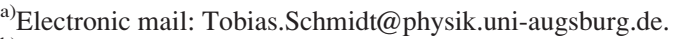

b)Electronic mail: Wolfgang.Bruetting@physik.uni-augsburg.de.
}

nantly horizontal orientation that strongly increases the efficiency of OLEDs. ${ }^{8}$ Implementing these oriented emitters promises a huge potential for improving the light outcoupling efficiency of OLEDs. ${ }^{6-8}$ In an optimized OLED stack, horizontal dipoles preferentially emit into air as compared to vertical emitters, ${ }^{8}$ resulting in a significant increase of the outcoupling factor $\eta_{\text {out }}$. Additionally, the knowledge of the emitter orientation is essential for a reliable efficiency analysis of OLEDs based on measured EQE data (see Eq. (1)). If the effect of orientation on $\eta_{\text {out }}$ is not taken into account properly, the determination of other factors in Eq. (1) like $\gamma$, $\eta_{\mathrm{s} / \mathrm{t}}$, or $q_{\mathrm{eff}}$ will be erroneous.

In this letter, we report on the red phosphorescent emitter Iridium(III)bis(2-methyldibenzo-[f,h]quinoxaline) (acetylacetonate) $\left(\operatorname{Ir}(\mathrm{MDQ})_{2}(\mathrm{acac})\right)$ doped with $8 \mathrm{wt} . \%$ in an $\mathrm{N}, \mathrm{N}^{\prime}$-bis(naphthalen-1-yl)-N, $\mathrm{N}^{\prime}$-bis(phenyl)benzidine $(\alpha-\mathrm{NPD})$ matrix in state-of-the art bottom-emitting OLEDs (structure shown in Fig. 1; for details of device fabrication, see Ref. 8). We will demonstrate that phosphorescence lifetime data for a series of devices with varying distance of the emission layer to the cathode can only be described consistently by optical simulations, if a non-isotropic orientation of the transition dipole moments of the emitting phosphorescent molecules is assumed, even though the $\alpha$-NPD matrix is optically isotropic. In contrast to recently reported results by Meerheim et al., ${ }^{9}$ we will furthermore show that the radiative quantum efficiency (RQE) extracted from lifetime data is overestimated, if the orientation of the emissive dipoles is not properly considered.

To determine the RQE of the emitter in the used guesthost system we investigate changes of the phosphorescence lifetime after optical excitation with a pulsed nitrogen laser (wavelength $337 \mathrm{~nm}$, pulse energy $10 \mu \mathrm{J}$, and pulse duration $750 \mathrm{ps}$ ). These measurements are performed on a series of OLEDs, where the distance of the emitter to the metallic mirror (cathode) is varied by changing the thickness of the electron transport layer (ETL) between 40 and $375 \mathrm{~nm}$. An exemplary phosphorescence decay curve together with a monoexponential fit can be seen in Fig. 1 . 


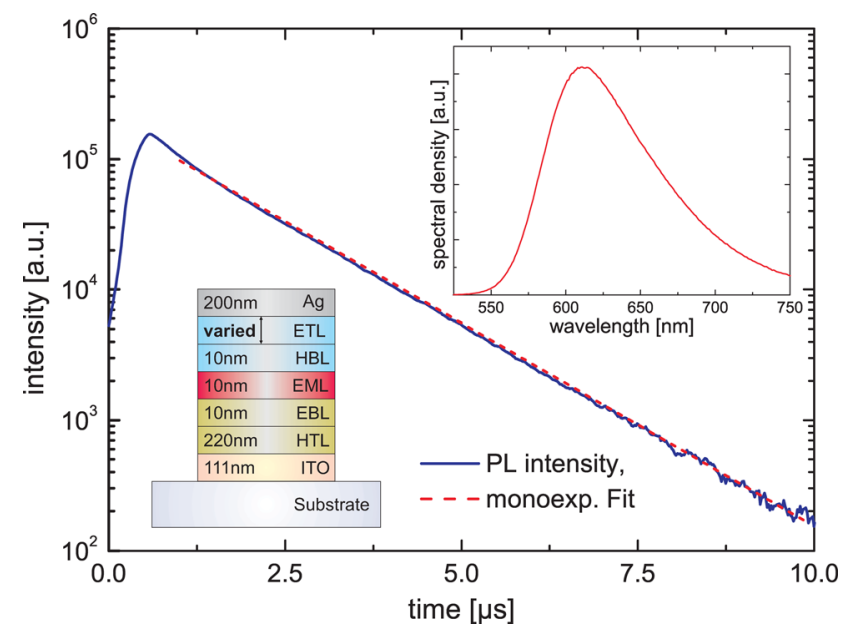

FIG. 1. (Color online) Layer stack of the investigated devices together with an exemplary phosphorescence decay (blue curve) and a monoexponential fit (dashed red line) yielding $\tau=1.36 \mu$ s for an ETL thickness of $249 \mathrm{~nm}$. The inset shows the intrinsic emitter spectrum used for optical simulations.

The obtained phosphorescence lifetimes for different ETL thicknesses shown in Fig. 2 are subsequently analyzed by numerical simulations as described in detail in Ref. 10 and as discussed in the following. The simulation, which is based on a dipole model and a transfer matrix formalism, ${ }^{11-13}$ evaluates the effect of the cavity on the radiative rate of the emitter. By this means the Purcell factor $F$ is calculated separately for each of the three orthogonal dipole orientations $(x, y$, and $z$ )

$$
F_{i}=\int_{\lambda_{1}}^{\lambda_{2}} S(\lambda) \int_{0}^{\infty} P_{i}\left(k_{\|}, \lambda\right) d k_{\|} d \lambda .
$$

Therein $S(\lambda)$ represents the intrinsic emitter spectrum (normalized to integral 1) shown as inset in Fig. 1 and the integration over the wavelength in Eq. (2) is performed over the whole spectral range of emission. Finally, $P_{i}\left(k_{\|}, \lambda\right)$ is the power dissipation spectrum parameterized with respect to the in-plane wave vector component $k_{\|}$and implicitly depending on the emitter/mirror distance, i.e., basically the ETL thickness, for each emitter orientation. Averaging over all emitter orientations by taking into account possible deviations from

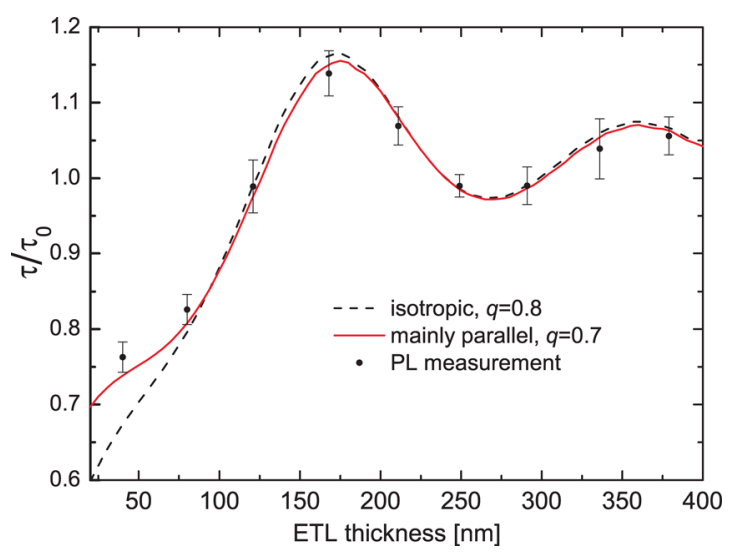

FIG. 2. (Color online) Measured phosphorescence lifetimes normalized to the intrinsic value $\tau_{0}=1.37 \mu$ s together with simulated curves for different emitter orientations as a function of the ETL thickness: isotropic with $\Theta=1 / 3$ and $q=0.8$ (dashed black line) and oriented with $\Theta=0.24$ and $q=0.7$ (solid red line). randomness through an orientation anisotropy factor $\Theta$ (measuring the relative content of vertically oriented dipoles with respect to the total number of dipoles, so that $\Theta=\frac{1}{3}$ for isotropic, 0 for completely horizontal (x-y-plane) and 1 for fully vertical orientation (z-direction)) yields the total emitter decay rate $\Gamma(\Theta)$ and thus the phosphorescence lifetime $\tau(\Theta)$ of excited states in a given OLED (Ref. 14)

$$
\tau(\Theta)^{-1}=\Gamma(\Theta)=\Gamma_{r} \cdot F(\Theta)+\Gamma_{n r} .
$$

Thereby $\Gamma_{r}$ and $\Gamma_{n r}$ are the radiative and the non-radiative intrinsic decay rates, respectively, and $F(\Theta)$ is the weighted sum of the three Purcell factors at each ETL thickness

$$
F(\Theta)=\frac{(1-\Theta)}{2}\left(F_{x}+F_{y}\right)+\Theta \cdot F_{z} .
$$

Finally, the relative change of the lifetime with respect to the value $\tau_{0}$ measured in the absence of the cavity $\left(F_{i} \equiv 1\right)$ is obtained by taking into account the radiative quantum efficiency of the emitter material $q=\Gamma_{r} /\left(\Gamma_{r}+\Gamma_{n r}\right)$

$$
\frac{\tau(\Theta)}{\tau_{0}}=\frac{\Gamma_{0}}{\Gamma(\Theta)}=[(1-q)+q \cdot F(\Theta)]^{-1} .
$$

Figure 2 shows a comparison of experimental lifetime data with simulations for two different emitter orientations and corresponding values of $q$. The black curve is the calculated relative lifetime that changes with the ETL thickness for a random emitter orientation $(\Theta=1 / 3)$ and an RQE of $80 \%$, while the red curve represents a non-isotropic, predominantly horizontal orientation with $\Theta=0.63 / 2.63=0.24$ taken from Ref. 8 and the corresponding RQE of $70 \%$. The emitter orientation of $\operatorname{Ir}(\mathrm{MDQ})_{2}$ (acac) in $\alpha$-NPD has previously been determined by two different methods as described in Refs. 5 and 15. Please note that the devices under investigation in the present study have been fabricated identically to the devices in Ref. 8. Comparison of both simulation results in Fig. 2 reveals that the assumption of non-isotropic emitter orientation (red curve) yields a slightly better fit of the measured phosphorescence lifetime data. All data are normalized with an intrinsic lifetime $\tau_{0}$, which is a fitting parameter of the simulations and can be determined by this comparison to $\tau_{0}=1.37 \mu \mathrm{s}$. In particular, for ETL thicknesses below $100 \mathrm{~nm}$ where the coupling of horizontally and vertically oriented dipoles to surface plasmons is markedly different, the non-isotropic simulation shows a much better agreement with the measured phosphorescence lifetimes. Moreover, the extracted value of the radiative quantum efficiency $q$ of $80 \%$ ( $\pm 5 \%$ ) for isotropic orientation, as proposed in Ref. 9, clearly overestimates the true value of about $70 \%$ $( \pm 5 \%)$ for the correct emitter orientation. This explicitly underlines the importance of knowing the dipole orientation of an emitter system to obtain valid results.

Another method to determine the RQE of an emitter/matrix system is the measurement of the external quantum efficiency under electrical operation of the OLEDs, e.g., with and without an outcoupling structure. ${ }^{9,16}$ As described in detail in Ref. 10, this method relies on the fact that the electrical characteristics are not affected by changing the thickness of the doped ETL. Figure 3 shows EQE measurements 


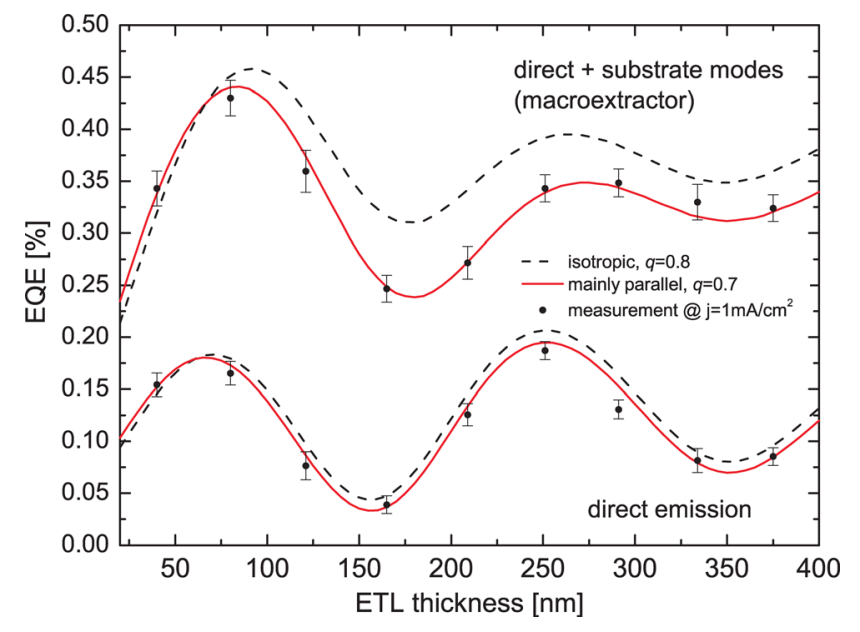

FIG. 3. (Color online) Measurements at a current density of $j=1 \mathrm{~mA} / \mathrm{cm}^{2}$ of the external quantum efficiency of OLEDs with different ETL thicknesses (without and with macroextracor) together with simulations assuming different emitter orientation: isotropic with $\Theta=1 / 3$ and $q=0.8$ (dashed black line) and oriented with $\Theta=0.24$ and $q=0.7$ (solid red line).

(without and with macroextractor) that have been performed in a calibrated integrating sphere for a current density of $j=1 \mathrm{~mA} / \mathrm{cm}^{2}$ together with numerical simulations of the EQE for the same emitter orientations as above. Please note that if a macroextractor is used both the direct emission and the substrate modes are extracted to air. In particular, the results obtained with a glass hemisphere applied to the substrate as macroextractor clearly demonstrates that a consistent description of the experimental data can only be achieved if the non-isotropic emitter orientation is regarded. Furthermore the best fit values of the RQE of $70 \%$ for the predominantly horizontal orientation and $80 \%$ for isotropic orientation are in excellent agreement with the phosphorescence lifetime analysis discussed above.

To demonstrate the enormous potential of oriented emitters in phosphorescent OLED structures, simulations for perfectly random and completely horizontal emitter orientations have been performed assuming an RQE of 100\%. Figs. 4(a) and 4(b) show the calculated power dissipation spectra for the stack under investigation in both cases. The simulations reveal an enhancement of the EQE by a factor of 1.4 if all of the substrate radiation could be extracted. Remarkably, $70 \%$ of the total internally emitted power could be coupled out to air in this case. If only the direct emission of the device is of interest, an enhancement by a factor 1.35 can be reached and about $38 \%$ of the power is emitted directly to air. This results mainly from the reduced coupling to surface plasmons for small ETL thicknesses and due to a less pronounced coupling to substrate and waveguided modes for higher ETL thicknesses.

In conclusion, we have demonstrated an essentially nonisotropic emitter orientation in a red phosphorescent OLED and discussed the consequences for a reliable determination of the radiative quantum efficiency of the emitter material. If the emitter orientation is not taken into account properly, the RQE or other quantities, like the singlet/triplet ratio, are significantly overestimated leading to wrong conclusions on the efficiency limiting factors of a given OLED structure. In addition we have demonstrated the huge potential of hori-

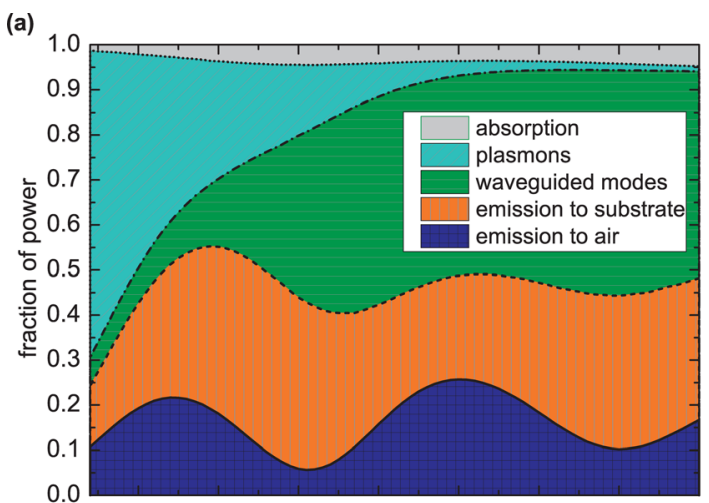

(b)

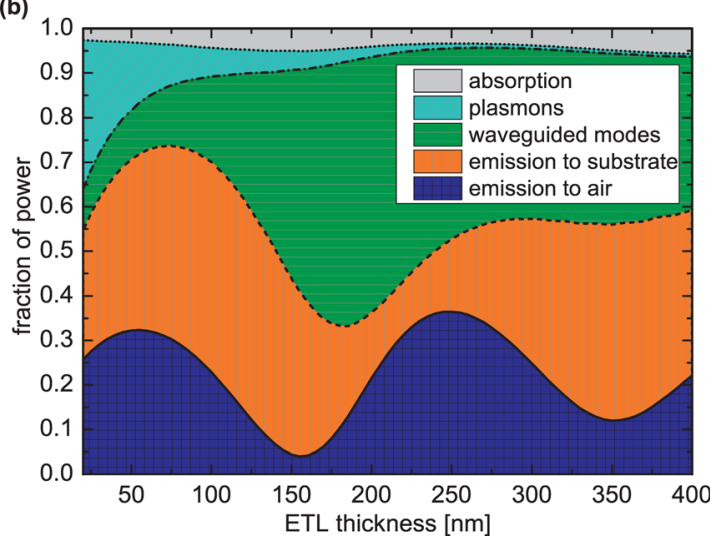

FIG. 4. (Color online) Calculated power distribution between different modes for the OLED stack depicted in Fig. 1 as a function of the ETL thickness for $q=1$. (a) isotropic $(\Theta=1 / 3)$ and (b) completely horizontal $(\Theta=0)$ emitter orientation.

zontal emitter orientation by numerical calculations predicting EQE values of up to $70 \%$.

We acknowledge financial support by the German Federal Ministry of Education and Research (BMBF) under the Contract No. FKZ 13N10474 (TOPAS 2012).

${ }^{1}$ T. Tsutsui, E. Aminaka, C. P. Lin, and D.-U. Kim, Philos. Trans. R. Soc. London, Ser. A 355, 801 (1997).

${ }^{2}$ S. Nowy, B. Krummacher, J. Frischeisen, N. Reinke, and W. Brütting, J. Appl. Phys. 104, 123109 (2008).

${ }^{3}$ W. L. Barnes, J. Mod. Opt. 45, 661 (1998).

${ }^{4}$ J. A. E. Wasey, A. Safonov, I. D. W. Samuel, and W. L. Barnes, Opt. Commun. 183, 109 (2000).

${ }^{5}$ M. Flämmich, M. C. Gather, N. Danz, D. Michaelis, A. H. Bräuer, K. Meerholz, and A. Tünnermann, Org. Electron. 11, 1039 (2010).

${ }^{6}$ D. Yokoyama, Y. Sakaguchi, M. Suzuki, and C. Adachi, Org. Electron. 10, 127 (2009).

${ }^{7}$ J. Frischeisen, D. Yokoyama, A. Endo, C. Adachi, and W. Brütting, Org. Electron. 12, 809 (2011).

${ }^{8}$ M. Flämmich, J. Frischeisen, D. S. Setz, D. Michaelis, B. C. Krummacher, T. D. Schmidt, W. Brütting, and N. Danz, Org. Electron. 12, 1663 (2011).

${ }^{9}$ R. Meerheim, M. Furno, S. Hofmann, B. Lüssem, and K. Leo, Appl. Phys. Lett. 97, 253308 (2010).

${ }^{10}$ D. S. Setz, T. D. Schmidt, M. Flämmich, S. Nowy, J. Frischeisen, B. C. Krummacher, T. Dobbertin, K. Heuser, D. Michaelis, N. Danz, W. Brütting, and A. Winnacker, J. Photon. Energy 1, 011006 (2011).

${ }^{11}$ R. R. Chance, A. Prock, and R. Silbey, Adv. Chem. Phys. 37, 1 (1978).

${ }^{12}$ J. A. E. Wasey and W. L. Barnes, J. Mod. Opt. 47, 725 (2000).

${ }^{13}$ N. Danz, R. Waldhäusl, A. Bräuer, and R. Kowarschik, J. Opt. Soc. Am. B: Opt. Phys. 19, 412 (2002).

${ }^{14}$ S. Mladenovski, S. Reineke, and K. Neyts, Opt. Lett. 34(9), 1375 (2009).

${ }^{15}$ J. Frischeisen, D. Yokoyama, C. Adachi, and W. Brütting, Appl. Phys. Lett. 96, 073302 (2010).

${ }^{16}$ B. Krummacher, S. Nowy, J. Frischeisen, M. Klein, and W. Brütting, Org. Electron. 10, 478 (2009). 